\title{
case report!
}

\section{Management of a fractured incisor using a stratilified}

composite technique

\section{Jatinder Heer ${ }^{1}$}

\section{Case report}

A 54-year-old female patient was referred for restoration of her fractured tooth (22). This tooth was traumatised several months earlier in a sports injury, and a composite restoration was provided. The referral was made with a view to crowning this tooth. The medical history was unremarkable. The patient sought an aesthetic functional restoration.

From the clinical appearance (Figs 1-2), the tooth was set in an anterior position to avoid the over-erupted lower incisor (32). This may be a result of the original restoration.

The 22 had been restored with a composite which was largely discoloured. The aesthetics of this restoration were unacceptable (Fig. 3).

The treatment proposed was to replace this restoration with a nano-composite (Filtek $\mathrm{XT}^{\mathrm{TM}}$ ). A stratified lamellar composite build up was proposed using three shades (A2B, A1E, WE).

A multi-shade composite is one where different composite layers are added in succession to provide a more lifelike end composite shade match in various light conditions. Often there are four layers and composite manufacturers will produce a shade guide to indicate which combinations should be used. The placements of these composites is also documented so that translucent, body, dentine and enamel shades are placed in the correct regions. Surgery Clinical Assistant, JH Dental Clinic, 279 Mitchell Ave,

Coventry, CV4 8DU

Email: jheer@talk21.com
General Dental Practitioner \& Oral

\section{Materials and methods}

Treatment was proposed under rubber dam isolation using Filtek XT due to its ease of use and the range of shades available. The kit is provided with a shade wheel which enables the operator to predict the end result. This material is also a nano-filled composite which provides a great deal of polishability. Guidelines are also provided for the layered method. A single, double or four stage method can be employed depending upon the amount of available space and aesthetic requirements.

Both the composite kit and the bonding resins are compatible and are provided with clear instructions on their use. The shade wheel in particular provides a quick reference for the practitioner.

Initially a temporary palatal composite was placed (Fig. 4). The occlusion was then reassessed and a putty record taken of the palatal surface (Fig. 5). This temporary composite was not bonded to the tooth.

Under local anaesthetic infiltration, the tooth was isolated using a medium rubber dam (Fig. 6). The adjacent teeth were protected with PTFE tape and the temporary composite removed. The enamel was bevelled buccally and all remnants of the original restoration removed.

A single step etch/bond system was used, Adper L-POP ${ }^{\mathrm{TM}}$ (Fig. 7). This is presented as a single use system which is mixed and applied to the tooth (15s) and allowed to gently air dry (3s) to enable the solvent to evaporate. The resin is then light cured (10s). This was repeated twice.

The enamel margin was bevelled extending towards the cervical margin. This greatly increases the bonding surface and reduces the line join that can sometimes be visible.

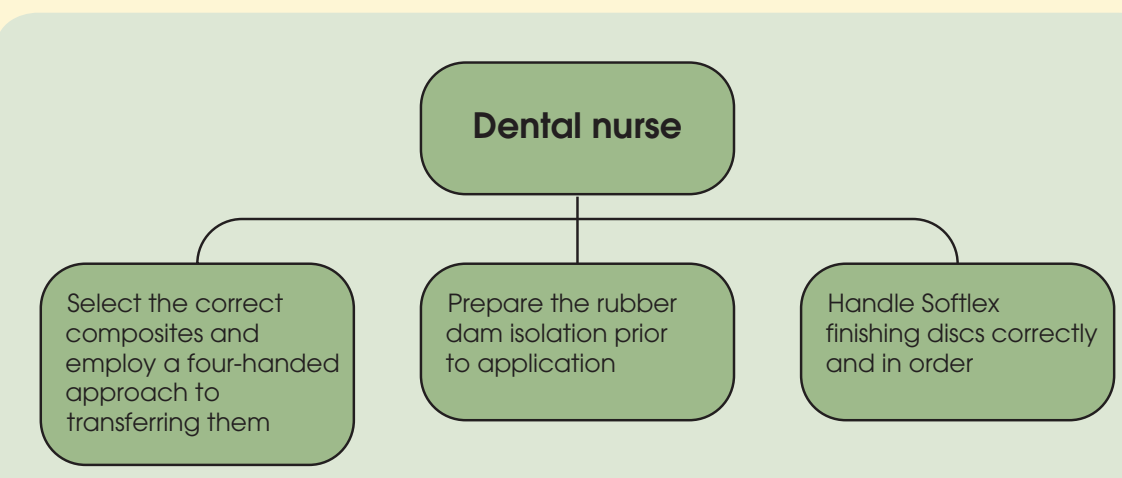

Close four-handed dentistry between the dentist and the dental nurse will make this procedure easier to perform and enable effective treatment while adhering to cross infection control protocol. This diagram summarises the dental nurse's contribution to this particular procedure. 
The putty record was then replaced (Fig. 8), and the first layer of composite placed to restore the palatal surface (Shade WE). The approximal surfaces were also restored at this stage and light cured (20s).

The dentine body was restored using A2 base recreating a mamelon structure internally. This was then veneered with A1E to replace the missing enamel and to provide the desired end result of A2. This technique is versatile and changes can be made to the shade mixtures to achieve an exact colour match. This would not be possible with a single shade technique.

Once light cured the tooth was contoured using tungsten carbide finishing burs and the correct sequence of four Softlex discs. Interproximal finishing Softlex strips were also used to hinder plaque stagnation approximally.

\section{Review}

Review appointments indicated that the restoration was well tolerated by the soft tissues and there were no detected adverse occlusal features. The nano-composite was able to retain its polish (Figs 9-11).

Historically composites consisted of mixed irregular sized filler particles. It was thought that the larger particles impart strength, while the small particles were able to produce a good degree of lustre. However, as the composites wear down with use, loss of the larger filler particles causes loss of polish/ lustre and hence this irregular surface scatters reflected light differently to the initial smooth surface.

Nano-composites have a far smaller particle size and the nano-clusters present within the composites are silane-coated. These materials will lose only smaller particles and hence have a better long term polish as the light reflection is far better long term. The nano-clusters help impart strength. The material used in this case was Filtek $\mathrm{XT}^{\mathrm{TM}}(3 \mathrm{M})$ for this reason as it is a true nano-composite.

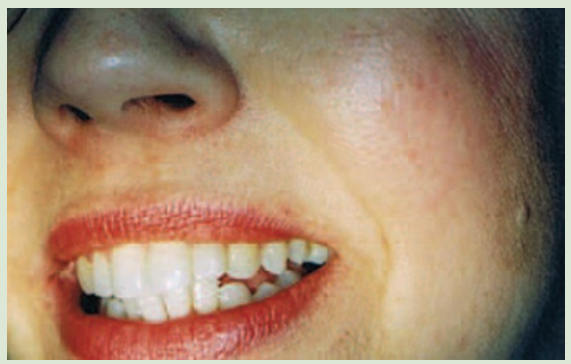

Fig. 9 Initial post-op view

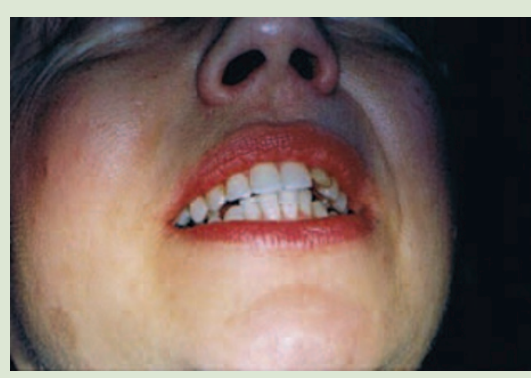

Fig. 1 Pre-op clinical appearance

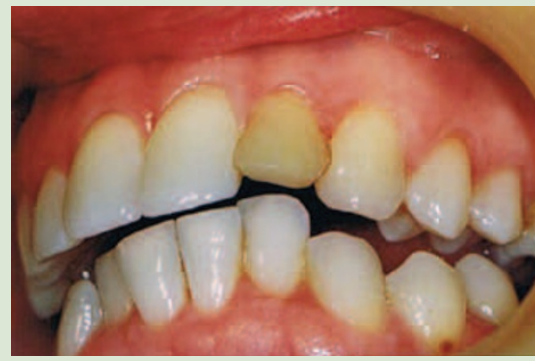

Fig. 3 Excessive proclination

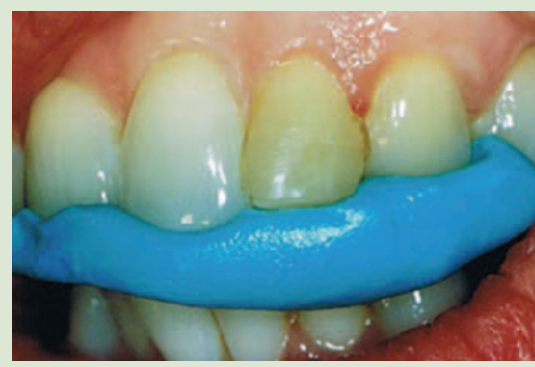

Fig. 5 Putty record of palatal contour

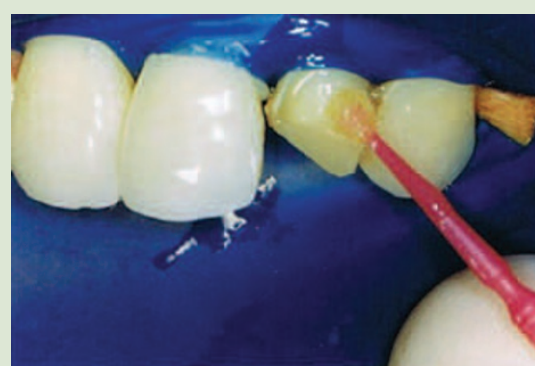

Fig. 7 Application of prime and bond (Adper L-POPTM)

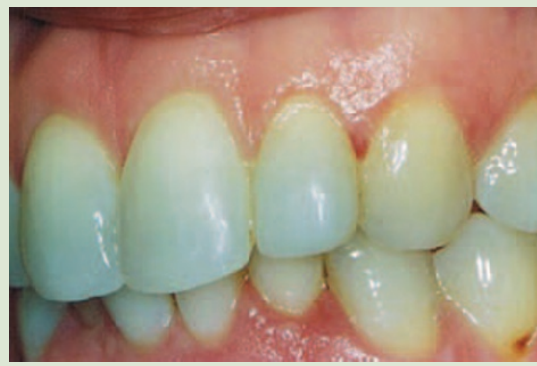

Fig. 10 Two weeks post-op

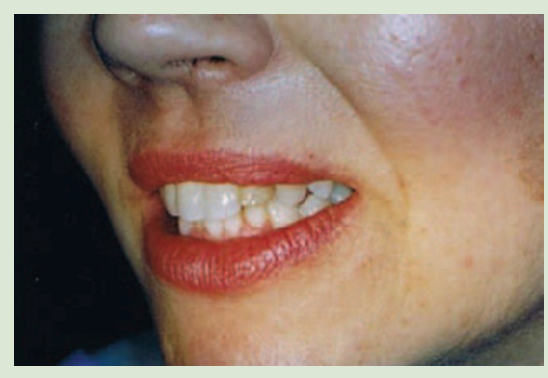

Fig. 2 Discoloured malpositioned

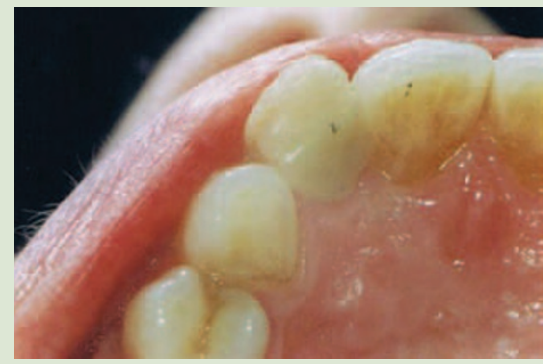

Fig. 4 Palatal form with temporary composite (mirror view)

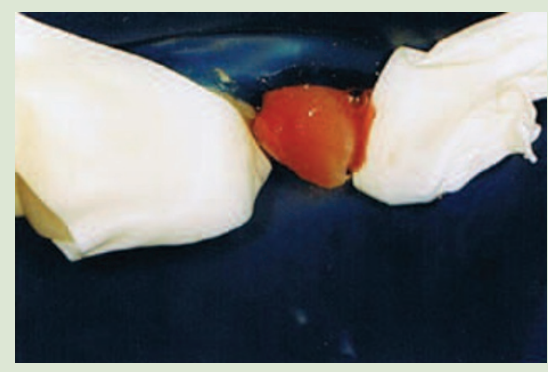

Fig. 6 Isolation and etching (Rubber dam and PTFE tape)

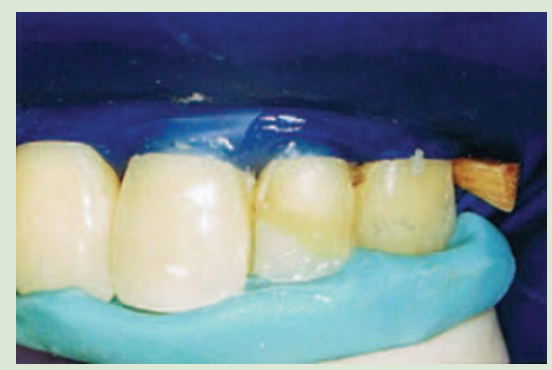

Fig. 8 Palatal composite layer

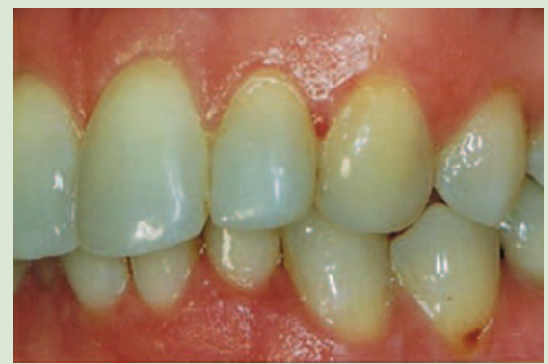

Fig. 11 Eight weeks post-op 